\title{
Multimodal optical measurement for study of lower limb tissue viability in patients with diabetes mellitus
}

Viktor V. Dremin

Evgeny A. Zherebtsov

Victor V. Sidorov

Alexander I. Krupatkin

Irina N. Makovik

Angelina I. Zherebtsova

Elena V. Zharkikh

Elena V. Potapova

Andrey V. Dunaev

Alexander A. Doronin

Alexander V. Bykov

Ilya E. Rafailov

Karina S. Litvinova

Sergei G. Sokolovski

Edik U. Rafailov 


\title{
Multimodal optical measurement for study of lower limb tissue viability in patients with diabetes mellitus
}

\author{
Viktor V. Dremin,, ${ }^{a, \star}$ Evgeny A. Zherebtsov, ${ }^{b}$ Victor V. Sidorov, ${ }^{c}$ Alexander I. Krupatkin, ${ }^{\text {d }}$ Irina N. Makovik, \\ Angelina I. Zherebtsova, ${ }^{\mathrm{a}}$ Elena V. Zharkikh, ${ }^{\mathrm{a}}$ Elena V. Potapova, ${ }^{\mathrm{a}}$ Andrey V. Dunaev, ${ }^{\mathrm{a}}$ Alexander A. Doronin, ${ }^{\mathrm{e}}$ \\ Alexander V. Bykov, ${ }^{f}$ Ilya E. Rafailov, ${ }^{\mathrm{g}}$ Karina S. Litvinova, ${ }^{\mathrm{h}}$ Sergei G. Sokolovski, ${ }^{\mathrm{b}}$ and Edik U. Rafailov ${ }^{\mathrm{b}}$ \\ ${ }^{a}$ Orel State University named after I.S. Turgenev, Biomedical Photonics Laboratory of University Clinic, Orel, Russia \\ ${ }^{\mathrm{b}}$ Aston University, Aston Institute of Photonic Technologies, Optoelectronics and Biomedical Photonics Group, Birmingham, United Kingdom \\ "SPE "LAZMA" Ltd., Moscow, Russia

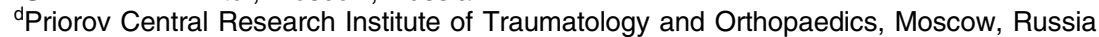 \\ eYale University, Department of Computer Science, Computer Graphics Group, New Haven, Connecticut, United States \\ fUniversity of Oulu, Optoelectronics and Measurement Techniques Laboratory, Faculty of Information Technology and Electrical Engineering, \\ Oulu, Finland \\ ${ }^{9}$ Aston University, School of Engineering and Applied Sciences, Aston Institute of Photonic Technologies, Birmingham, United Kingdom \\ ${ }^{\mathrm{h}}$ Aston University, Aston Medical School, Birmingham, United Kingdom
}

\begin{abstract}
According to the International Diabetes Federation, the challenge of early stage diagnosis and treatment effectiveness monitoring in diabetes is currently one of the highest priorities in modern healthcare. The potential of combined measurements of skin fluorescence and blood perfusion by the laser Doppler flowmetry method in diagnostics of low limb diabetes complications was evaluated. Using Monte Carlo probabilistic modeling, the diagnostic volume and depth of the diagnosis were evaluated. The experimental study involved 76 patients with type 2 diabetes mellitus. These patients were divided into two groups depending on the degree of complications. The control group consisted of 48 healthy volunteers. The local thermal stimulation was selected as a stimulus on the blood microcirculation system. The experimental studies have shown that diabetic patients have elevated values of normalized fluorescence amplitudes, as well as a lower perfusion response to local heating. In the group of people with diabetes with trophic ulcers, these parameters also significantly differ from the control and diabetes only groups. Thus, the intensity of skin fluorescence and level of tissue blood perfusion can act as markers for various degrees of complications from the beginning of diabetes to the formation of trophic ulcers. @ 2017 Society of Photo-Optical Instrumentation Engineers (SPIE) [DOI: 10.1117/1.JBO.22.8.085003]
\end{abstract}

Keywords: fluorescence spectroscopy; metabolism; advanced glycation end products; mitochondrial function; laser Doppler flowmetry; diabetes mellitus; diagnostic volume.

Paper 170281RR received Apr. 28, 2017; accepted for publication Jul. 21, 2017; published online Aug. 19, 2017.

\section{Introduction}

According to the International Diabetes Federation (IDF), the challenge of early stage diagnosis and monitoring of the treatment effectiveness in diabetes is currently one of the highest priorities in modern healthcare. The medical, social, and economic significance of diabetes is primarily determined by the high prevalence of this disease and the frequency of debilitating and quality of life-reducing effects suffered by affected individuals. The IDF report for 2015 indicated that there are 415 million diabetic patients worldwide, with this figure projected to grow to 642 million by $2040 .{ }^{1}$

Over the last several years, multiple studies have indicated that a timely diagnosis and treatment, including an increased level of patient monitoring, reduce the manifestation of various complications, potentially even reversing them at early preclinical stages. Particular attention is given to foot complications such as peripheral arterial disease (PAD), neuropathy, and skin disorders including foot ulcers, which are liable for more hospitalizations than any other complication of diabetes. ${ }^{2}$ Particularly of interest, however, are vascular disorders such as PAD, which lead to the most adverse implications for patients. Macro-

*Address all correspondence to: Viktor V. Dremin, E-mail: dremin_viktor@mail.ru and microcirculatory disturbances, which are often imperceptibly formed, significantly reduce the patient's life quality. This can eventually lead to an early death of the patient. Disorders of microcirculation can occur long before the clinical manifestation of diabetes and play a central role in the development of foot ulcers and their subsequent inability to heal. ${ }^{3-7}$

Available diagnostic approaches for the investigation of PAD at the level of macro- and microcirculation offer both advantages and disadvantages inherent to each method. Currently, the commonly employed standards of diabetic foot disorder detection in hospitals or surgery are predominantly thorough medical history examination, visual inspection, and duplex ultrasonography scanning of arteries. The latter methods are capable of determining the level of trophic disorders, specifically the hemodynamics. However, for microcirculatory disturbances, ultrasound Doppler is not efficient enough. ${ }^{8}$ Other methods such as radiocontrast angiography have significant limitations during regular patient control: invasiveness, contrast agent toxicity, and prolonged exposure of the patient to radiation. ${ }^{9,10}$ In addition to the methods, medical device manufacturers offer solutions that allow for a joint diagnosis of macrovascular [measurement of ankle brachial index, toe brachial index, pulse volume recording, and 
segmental pressure (SP) with measurement under ultrasonic or laser Doppler flowmetry control $]^{11,12}$ and microvascular (transcutaneous oximetry) disorders. Often, this allows the acquisition of qualitative information about PADs and assessment of the wound-healing potential. However, the interpretation of the described parameters of vascular disorders can be somewhat subjective. The measurement results are affected by arterial calcifications as well as the rigidity of the vascular wall. ${ }^{13-15}$ Also, it is known that these methods can underestimate the true prevalence of cardiovascular diseases and, in particular, do not detect an early phase of vascular complications. ${ }^{16}$ The method of transcutaneous oximetry $\left(\mathrm{TcpO}_{2}\right)$ allows assessment of the oxygen partial pressure in the intercellular liquids. ${ }^{17,18}$ Due to physiological, methodological, and technical obstacles, this approach was not widely used in polyclinic diagnostics. At the same time, the trustworthiness of data obtained by this method could be questioned because of low selectivity to infectious inflammation on the foot, peripheral edema, and other accompanying pathologies. ${ }^{19,20}$ Another conventionally employed approach is thermal imaging, which reveals thermal asymmetry zones on the limb surface. The result of the diagnostics, however, is usually meaningful only during late stages of the limb angiopathy, when the blood circulation is damaged on the level of great vessels. $^{21,22}$

Additionally, to date, a general drawback of the methods available in the physician's arsenal is the inability to monitor the metabolic processes occurring in biological tissue. Patients with diabetes often display severe disorders of microcirculation, accompanied by significant metabolic shifts. Consequently, oxygen transport, its delivery, and tissue consumption are the most important indicators of the functioning of life support systems, and their adequate evaluation is important for choosing the optimal methods and tactics for treating each individual patient.

Despite this, there is little information on the effect of changes in blood flow in the delivery of oxygen and tissue metabolism of the lower limbs in diabetes mellitus. The influence of the development of diabetes on foot metabolism has not been practically investigated at present. ${ }^{4}$ The main purpose of this article was to show a possible approach in assessing not only vascular disorders occurring in diabetes mellitus but also associated metabolic disorders.

It is known that variations in the respiratory chain enzyme activities are recognized as one of the consequences resulting from a breach in the cell function observable in diabetes mellitus. ${ }^{23-25}$ Currently, a lot of research is being done to study the metabolism of epithelial tissues, including skin, using fluorescence spectroscopy and imaging techniques. ${ }^{26-30}$ Changes in the mitochondrial respiratory chain are recognized as one of the consequences resulting from a breach in the cell function observable in different pathologies. The most likely changes to occur are the accumulation of a coenzyme nicotinamide adenine dinucleotide (NADH) (the reduced form) and flavin adenine dinucleotide (FAD) (oxidized form). Detection of such changes by fluorescence spectroscopy is one of the most promising directions for in vivo diagnosis.

A recent review $^{31,32}$ and some other studies show that changes in the fluorescence of the NADH respiratory chain were measured in single cells, ${ }^{33,34}$ tissue slices,${ }^{35}$ and organs. ${ }^{36-39}$ Despite the fact that the study of NADH and FAD fluorescence has been successfully held for the past 50 years, relatively little research has been conducted at the organ level. Meanwhile, the results of experiments on a particular organ or area of the body can be more successfully extrapolated to clinical practice in comparison to the studies at the cellular and subcellular levels.

Furthermore, it has been established in recent years that longexisting diabetes with hyperglycemia results in an increase in protein glycation levels. This is accompanied by an increase in so-called advanced glycation end products (AGEs), which influence the properties of collagen and other structural proteins of the capillary membrane and skin. ${ }^{40-43}$ Among a number of AGE (e.g., $\mathrm{N} \varepsilon$-carboxymethyllysine, $\mathrm{N} \varepsilon$-carboxyethyllysine, and pyrraline), pentosidine, responsible for the formation of adhesions between collagen fibers, has its own fluorescence. ${ }^{44-46}$

Thus, the development of new optical diagnostic methods in these latter days has potential to find promising application in diagnosing diabetes disorders. The advantage of these methods lies in their noninvasive properties, good spatial resolution, low cost procedures, and high productivity. One of the promising directions of optical diagnostics development is the establishment of a scientific and instrumental framework to create rapid and real-time in vivo analysis methods for determining biological tissue viability. ${ }^{47}$

For a comprehensive diagnosis of the state of microcirculatory-tissue systems, applying several diagnostic technologies simultaneously in one diagnostic volume is currently promising. ${ }^{31,48,49}$ To assess the state of blood flow and detect possible vascular disorders, different combinations of methods are applied. For instance, simultaneous measurements of the intensities of fluorescence and Rayleigh components in human eye lenses allow a good accuracy to separate patients with diabetes from a control group. ${ }^{50}$ Furthermore, diffuse reflectance spectroscopy can be used for correction of the data obtained during fluorescence studies. ${ }^{51,52}$ Another example of combined technologies is a lightguide tissue spectrophotometry $\left(\mathrm{O}_{2} \mathrm{C}\right)$. It is a combination of LDF and tissue spectrometry. This method allows for the measurement of both relative blood flow by the laser Doppler technique and hemoglobin oxygenation and hemoglobin amount in tissue by spectrometric techniques. ${ }^{53}$

In this study, we experimentally estimate the potential of coregistering cutaneous blood flow parameters and the intrinsic tissue fluorophore fluorescence to distinguish stages of the complications of lower extremities in patients with diabetes mellitus. From the point of view of the fluorescence analysis, it is logical to consider a complex parameter, which describes the oxidative metabolic processes (changing the contents of NADH and FAD) and the carbohydrate metabolism (AGEs accumulation) in tissue, related to patients with diabetes manifesting as complications of the lower limbs.

\section{Material and Methods}

\subsection{Experimental Equipment}

In the experimental studies, the tissue blood perfusion and the tissue fluorescence were assessed by LDF and FS, respectively. ${ }^{54}$ Registered perfusion parameters and the amplitude of the coenzymes' fluorescence were simultaneously evaluated using a specially designed system in the same tissue volume (SPE "LAZMA" Ltd., Russia). The Doppler channel built on the basis of the single-mode laser module used a wavelength of $1064 \mathrm{~nm}$. A 365-nm and 450-nm radiation sources were used for fluorescence excitation. A multioptical fiber probe was used for delivery of penetrating radiation and registration of backreflected secondary radiation from the tissue [Fig. 1(a)]. The probing fiber of the LDF channel has a diameter of $6 \mu \mathrm{m}$ and the 

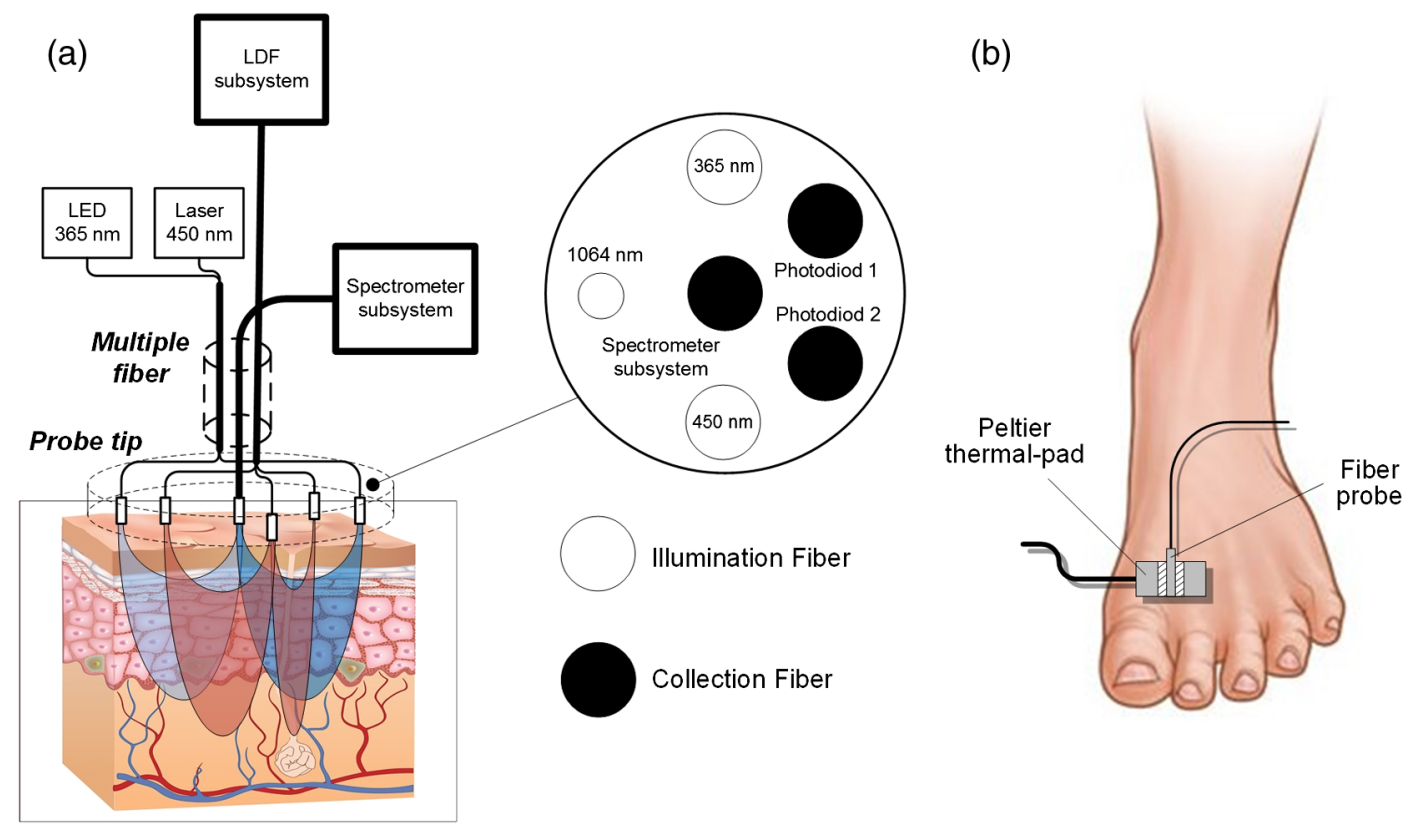

Fig. 1 (a) The general scheme of the measuring system and (b) localization of the fiber optic probe with Peltier thermal-pad.

receiving fiber has a diameter of $400 \mu \mathrm{m} .{ }^{55}$ The source-detector spacing for the LDF channel is $1.5 \mathrm{~mm}$. In the FS channel, diameters of all probing and receiving fibers are $400 \mu \mathrm{m}$. For safety reasons, as well as to keep photobleaching of the tissue at an acceptable level, probe radiation power of the 365 -nm excitation wavelength at the output of the fiber probe did not exceed $1.5 \mathrm{~mW}$. To assess the safety of the probe, coupled with the LED, we used documentation of the International Commission on Nonionizing Radiation Protection (ICNIRP). ${ }^{56}$ The calculation gives the effective skin irradiance of $1.4 \mathrm{~W} / \mathrm{m}^{2}$. According to the cited guideline document, the limiting UV exposure duration per day for the level of effective irradiance is at least $10 \mathrm{~s}$. During this protocol, the actual time of the UV-irradiation did not exceed $1 \mathrm{~s}$. Therefore, from the point of view of the ICNIRP recommendations, the proposed protocol of using the probe was deemed to be safe. The output power for the 450-nm excitation wavelength did not exceed $3.5 \mathrm{~mW}$. The source-detector spacing for the FS channel was $1 \mathrm{~mm}$. The numerical aperture of the fibers was 0.22 .

Specialized software was developed to work with the system. This allowed for real-time control of the course of the experiment and analysis of the recorded parameters. It further allowed the control of the unit responsible for realizing thermal functional tests.

\subsection{Experimental Study}

The experimental study involved 76 patients with Caucasian skin type and with type 2 diabetes mellitus at the Endocrinology Department of the Orel Regional Clinical Hospital (Orel, Russia). The study did not include volunteers with cardiovascular, bronchopulmonary, or neuroendocrine system disease comorbidities, nor with diseases of the gastrointestinal tract, liver, kidneys, blood, and any other serious chronic diseases, which could have an impact on the diagnostic results. Volunteers with a history of alcoholism, drug addiction, and drug abuse were also excluded. Laboratory parameters were measured according to standard laboratory procedures. The measurement of blood pressure was carried out after 5 min of rest, with the patient in a sitting position. Fourteen people from the patient's group were considered to have a more severe course of the disease (diabetic group 2). The decision on the degree of severity in each case was taken based on the presence of trophic disorders in the form of ulcers, analysis of anamnesis, and consultation with the attending physician. Thus, diabetic group 2 consisted of all diabetics with the presence of visible trophic ulcers, and diabetic group 1 included patients without trophic ulcers. Patients were examined on the first day of admission to the clinic. Prior to hospitalization, patients took medication to reduce high blood glucose levels. The control group consisted of 48 healthy volunteers (mean age $46 \pm 6$ years). The participating volunteer group was collected through an internal university advertisement circulated by physical posters and notification e-mail. The experiments were conducted under established protocols. The study protocol was approved at a meeting of the ethics committee at Orel State University named after I.S. Turgenev from March 11, 2015 (minutes of the meeting No. 7). After receiving the description of the protocol, all participating volunteers signed an informed consent form.

The main characteristics of the studied groups are presented in Table 1 . With a specified probability of 0.01 , we can consider that the compared groups are statistically homogeneous with a difference in fasting glucose level and body mass index. The table shows that diabetic group 2 had a longer duration of diabetes and higher values of creatinine and urea (which may indicate kidney function is decreased), but these parameters did not reach statistically significant levels $(p<0.01)$. However, the percentage of complications in this group (diabetic polyneuropathy, diabetic retinopathy, diabetic nephropathy, and diabetic microangiopathy of the lower limbs) is statistically significant compared to diabetic group 1.

The local heating stimulation was selected as a provocative action on the blood microcirculation system, allowing assessment of the local regulatory mechanisms of blood flow. Using a special 
Table 1 Statistical difference between patient groups and data for controls.

\begin{tabular}{|c|c|c|c|c|}
\hline Characteristics & Diabetic group 1 & Diabetic group 2 & Control group & $P$ value \\
\hline $\operatorname{Sex}(M / F)$ & $20 / 42$ & $8 / 6$ & $30 / 18$ & 0.08 \\
\hline Age $(y)$ & $54 \pm 10$ & $53 \pm 13$ & $46 \pm 6$ & 0.89 \\
\hline Systolic BP (mmHg) & $136 \pm 16$ & $144 \pm 22$ & $128 \pm 9$ & 0.29 \\
\hline Diastolic BP (mmHg) & $83 \pm 8$ & $85 \pm 8$ & $80 \pm 5$ & 0.12 \\
\hline Body mass index $\left(\mathrm{kg} / \mathrm{m}^{2}\right)$ & $31.9 \pm 6.3$ & $32.0 \pm 6.2$ & $24.2 \pm 2.5$ & $<0.001^{a}$ \\
\hline Fasting glucose $(\mathrm{mmol} / \mathrm{l})$ & $9.3 \pm 4.8$ & $9.1 \pm 2.5$ & $4.8 \pm 0.4$ & $<0.001^{a}$ \\
\hline Diabetes duration $(\mathrm{y})$ & $11 \pm 7$ & $18 \pm 12$ & - & $0.03^{b}$ \\
\hline $\mathrm{HbA1c}(\%)$ & $8.6 \pm 1.5$ & $8.6 \pm 0.7$ & - & $0.28^{\mathrm{b}}$ \\
\hline $\mathrm{HbA} 1 \mathrm{c}(\mathrm{mmol} / \mathrm{mol})$ & $70 \pm 9$ & $70 \pm 4$ & - & $0.31^{\mathrm{b}}$ \\
\hline Total cholesterol $(\mathrm{mmol} / \mathrm{l})$ & $4.9 \pm 1.2$ & $5.1 \pm 1.3$ & - & $0.76^{\mathrm{b}}$ \\
\hline Creatinine $(\mu \mathrm{mol} / \mathrm{l})$ & $81.5 \pm 28.7$ & $90.3 \pm 16.9$ & - & $0.02^{\mathrm{b}}$ \\
\hline Urea (mmol/l) & $7.7 \pm 6.8$ & $8.2 \pm 2.3$ & & $0.09^{b}$ \\
\hline ALT (IU/L) & $34.2 \pm 20.3$ & $28.4 \pm 19.3$ & & $0.25^{\mathrm{b}}$ \\
\hline AST (IU/L) & $31.0 \pm 24.8$ & $24.1 \pm 13.4$ & & $0.13^{b}$ \\
\hline The presence of complications (\% of the total number of patients) & 15 & 50 & - & $0.007^{\mathrm{b}}$ \\
\hline
\end{tabular}

Note: Data are mean \pm SD unless stated otherwise.

Note: Reference values of the laboratory: $\mathrm{HbA} 1 \mathrm{c} 4.0 \%$ to $6.0 \%$, total cholesterol 3.5 to $5.0 \mathrm{mmol} / \mathrm{l}$, urea 2.5 to $7.5 \mathrm{mmol} / \mathrm{l}$, creatinine 70 to $110 \mu \mathrm{mol} / \mathrm{I}$, ALT 10 to $38 \mathrm{IU} / \mathrm{L}$, and AST 10 to $40 \mathrm{IU} / \mathrm{L}$.

${ }^{\mathrm{a} C}$ Control group versus diabetic group 1 and diabetic group 2 .

bDiabetic group 1 versus diabetic group 2. Nonparametric data of the three studied groups were analyzed by a Kruskal-Wallis analysis of variance (ANOVA). A Mann-Whitney U-test was used to identify differences between the two groups.

block "LAZMA-TEST" (SPE "LAZMA” Ltd., Russia), consecutive local cold $\left(25^{\circ} \mathrm{C}\right)$ and heat $\left(35^{\circ} \mathrm{C}\right.$ and $\left.42^{\circ} \mathrm{C}\right)$ provocations were applied for 4 min each. The first stage included registration of microcirculatory parameters in the basal conditions for a 4-min period and the recording of a pair of fluorescence spectra. Thus, the duration of one study was $\sim 16 \mathrm{~min}$.

In this study, the probe was secured on the dorsal surface of the foot to a point located on a plateau between the first and second metatarsal bones [Fig. 1(b)]. We investigated the area of nonglabrous skin, where changes in blood flow are mediated by the vessels themselves and by two mechanisms of the sympathetic nervous system: noradrenergic vasoconstrictor nerves and a cholinergic active vasodilator system. ${ }^{57}$ It is due to this that the bulk of the literature ${ }^{58,59}$ is focused on controls of the nonglabrous skin with less focus on the glabrous skin of the palms, soles, and digits. Moreover, some research stated that perfusion in the sites with arteriovenous anastomoses (with glabrous or nonhairy skin) may fluctuate substantially, ${ }^{60,61}$ so the microcirculation assessment in this area may not be a very sensitive indicator of disease severity. ${ }^{62}$ Particular attention was paid to minimize local pressure of the probe on skin, as the parameter strongly influences the measurement results of skin blood perfusion and fluorescence intensity. ${ }^{63}$ All studies were performed in the supine position. The volunteers were asked to refrain from food and drink $2 \mathrm{~h}$ before the study to exclude the influence of these factors on the change of microhemocirculation and metabolic processes. Room temperature was maintained at a steady $24^{\circ} \mathrm{C}$ to $25^{\circ} \mathrm{C}$. All volunteers underwent preliminary adaptation to these conditions for at least $10 \mathrm{~min}$. Furthermore, skin temperature change of volunteers was recorded during the study. In the basal conditions, subjects had different skin temperatures. In this regard, to unify measurements, we precooled the study area on the foot to $25^{\circ} \mathrm{C}$.

\subsection{Validation of Diagnostic Depth of the Probe}

Proper evaluation of fluorescence intensity measurements in healthy tissue and areas of pathology will be possible if the penetration depth of the radiation, as well as diagnostic volume, was known. It is also important for the researcher or practicing physician to understand the depth of penetration of probing radiation in laser Doppler measurements for a specific measuring device. ${ }^{64}$ This would provide information on the blood vessels involved in the formation of the signal.

Due to the complexity of the actual sensing conditions, as well as a complex composite structure of human skin, it is not possible to obtain a general analytical solution to evaluate the diagnostic volume. In this regard, the method of stochastic Monte Carlo (MC) simulations was used. Currently, there are some known realizations of this approach used to simulate the propagation of optical radiation in organic tissues. ${ }^{65-67}$ One of the more advanced approaches is the use of an object-oriented 

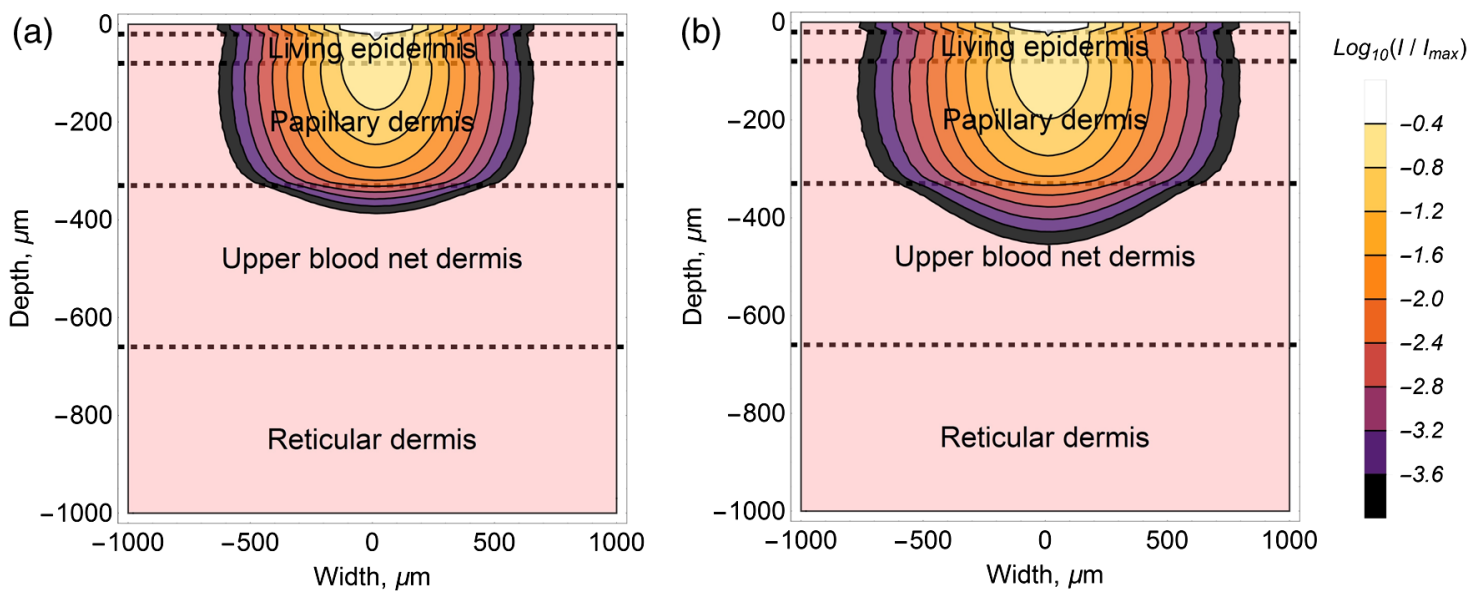

Fig. 2 Result of the modeling of diagnostic volume for the fluorescence measurements for the excitation wavelength of (a) 365 and (b) $450 \mathrm{~nm}$ for the parameters of the used optical fiber probe: the distance between the fibers: $1 \mathrm{~mm}$, fiber diameter: $400 \mu \mathrm{m}$, receiving aperture angle: 0.22 .

MC model, ${ }^{68-71}$ which allows for the description of the photons and the structural components of the tissue as independent objects interacting with each other. For acceleration of simulation performance, parallel computing was implemented on the used algorithms. Parallel computing technology CUDA (Computer Unified Device Architecture, NVIDIA Corporation) was employed as a framework of the calculations.

For both the LDF measurements and the fluorescence measurements, the diagnostic volume was assessed through an evaluation of the probe radiation distribution. For fluorescence measurements, the validity of this approach is based on the fact that, according to the rule of Stokes shift, the fluorescence spectrum is shifted into the long-wave region. It is also known that the absorption of light in most of the biological tissues for wavelengths of visible spectrum falls as wavelength increases. Thus, to get a lower-bound estimate of the diagnostic volume of the measurements, assessing the sampling volume of the radiation for a wavelength of fluorescence excitation is sufficient. One of the possible ways to formalize calculation of the sampling volume is calculating the distribution of rays reaching the detector using the MC method.

A seven-layer tissue model was used to simulate the diagnostic volume and depth of penetration of the probe radiation. This model was originally proposed in an earlier article. ${ }^{68}$ Figure 2(a) shows an example of the distribution obtained for the above simulation parameters for a wavelength of $365 \mathrm{~nm}$. Thus, diagnostic depth of the conducted diagnostics is at least several hundred microns and includes the epidermis, papillary dermis layer, and small part of upper blood net dermis [Fig. 2(a)]. Diagnostic volume, estimated by simulation, is in the order of $0.2 \mathrm{~mm}^{3}$.

For an excitation wavelength of $450 \mathrm{~nm}$, diagnostic depth reaches 450 to $500 \mu \mathrm{m}$ and penetrates the epidermis, papillary dermis, and most of the upper blood net dermis [Fig. 2(b)]. In this case, the diagnostic volume is approximately estimated by the simulation as $0.35 \mathrm{~mm}^{3}$.

The performed simulation shows that the proposed probe allows for the registration of fluorescence in the epidermis, mainly contributed to by NADH and FAD, and the dermis with a significant contribution from collagen.

The results of diagnostic volume modeling for the LDF probe show that the value is about $1.8 \mathrm{~mm}^{3}$. This confirms that this probe of the developed device is highly sensitive to the variations of blood flows in the papillary dermis and upper blood net plexus and is able to cover the top part of the reticular dermis (Fig. 3).

\subsection{Data Analysis}

The recorded and subsequently averaged amplitudes $\mathrm{AF}_{460}$ of the fluorescence at $460 \pm 10 \mathrm{~nm}$ with excitation at $365 \mathrm{~nm}$ and $\mathrm{AF}_{525}$ of the fluorescence at $525 \pm 10 \mathrm{~nm}$ with excitation at $450 \mathrm{~nm}$ (normalized to the intensity of the backscattered excitation radiation), as well as the average blood tissue perfusion $I_{m}$ at each stage of the functional test, were analyzed. The wavelengths were selected to maximize the probability of detection of NADH and FAD signals. Taking into account the relatively small sample sizes, nonparametric methods were used to confirm the reliability of differences in the results even in small groups of observations, namely the Mann-Whitney U-test and Kruskal-Walles ANOVA test. Values of $p<0.01$ were considered significant.

Considering the former division of experimental data into classes (two patient and one control), a linear discriminant

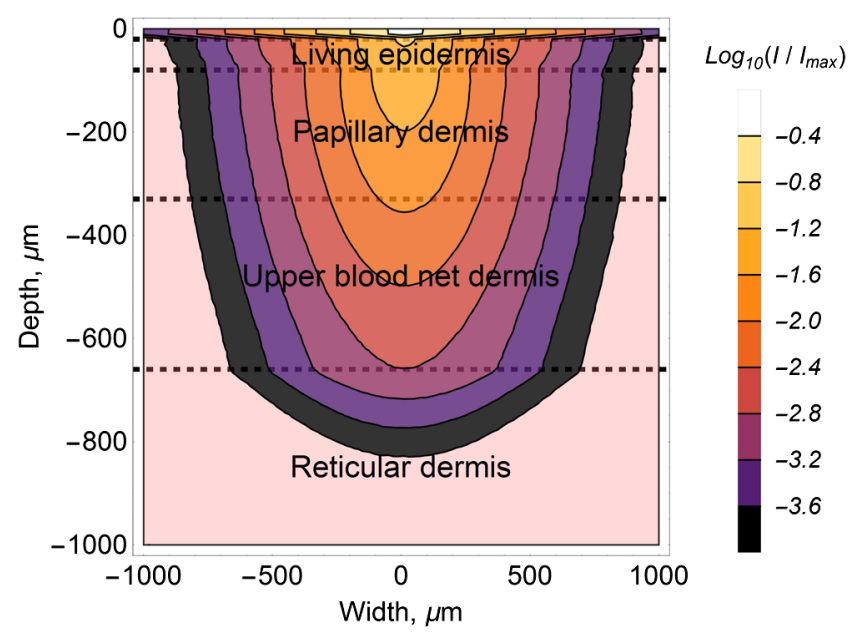

Fig. 3 The result of modeling the diagnostic volume for the LDF measurements for the wavelength of $1064 \mathrm{~nm}$, using the following optical fiber probe parameters: distance between the fibers: $1.5 \mathrm{~mm}$ and receiving aperture angle: 0.22 . 
Table 2 The results of experimental studies.

\begin{tabular}{lccc}
\hline Parameter & $\begin{array}{c}\text { Control } \\
\text { group }\end{array}$ & $\begin{array}{c}\text { Diabetic } \\
\text { group 1 }\end{array}$ & $\begin{array}{c}\text { Diabetic } \\
\text { group 2 }\end{array}$ \\
\hline $\begin{array}{l}\text { Fluorescence amplitude } \\
\mathrm{AF}_{460}, \mathrm{AU}\end{array}$ & $2.1 \pm 0.8$ & $2.7 \pm 0.8^{*}$ & $3.8 \pm 0.7^{* *}$ \\
$\begin{array}{l}\text { Fluorescence amplitude } \\
\mathrm{AF}_{525}, \mathrm{AU}\end{array}$ & $1.2 \pm 0.4$ & $1.8 \pm 0.7^{*}$ & $2.5 \pm 0.6^{* *}$ \\
Perfusion $I_{m}, \mathrm{PU}$ & $5.0 \pm 1.9$ & $5.3 \pm 2.2$ & $3.8 \pm 1.6$ \\
Perfusion $I_{m}^{25^{\circ} \mathrm{C}}, \mathrm{PU}$ & $4.4 \pm 1.6$ & $4.7 \pm 2.0$ & $3.6 \pm 1.4$ \\
Perfusion $I_{m}^{35^{\circ} \mathrm{C}}, \mathrm{PU}$ & $8.7 \pm 3.1$ & $6.6 \pm 2.4^{*}$ & $5.0 \pm 1.4^{* *}$ \\
Perfusion $I_{m}^{42^{\circ} \mathrm{C}}, \mathrm{PU}$ & $19.9 \pm 4.6$ & $12.3 \pm 3.5^{\star}$ & $9.2 \pm 4.6^{* *}$ \\
\hline
\end{tabular}

"Confirmed statistically significant differences between control group and diabetic group $1(p<0.01)$.

${ }^{* *}$ Confirmed statistically significant differences between diabetic group

1 and diabetic group $2(p<0.01)$.

analysis was employed to determine the discriminant function, leading to the synthesis of the desired decision rule. The obtained classifiers allow for a reappearing object to be assigned to one of the above classes by measured parameter values. Evaluation of the discriminant analysis quality was performed using the receiver operating characteristic (ROC) curve.

\section{Experimental Results and Discussion}

\subsection{Experimental Study}

Experimental studies have shown that diabetic patients have elevated values of normalized fluorescence amplitudes, as well as a lower perfusion response to local heating (up to $35^{\circ} \mathrm{C}$ and $42^{\circ} \mathrm{C}$ ). At the same time, in the group of diabetic patients with trophic ulcers (diabetic group 2), these parameters also significantly differ from the control and diabetes only groups (Table 2, Fig. 4).

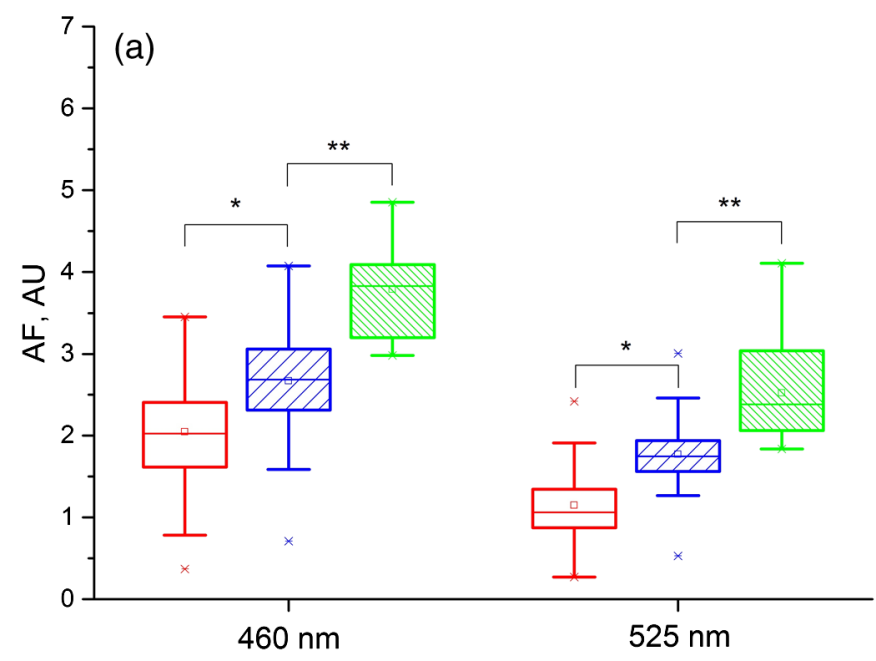

As already mentioned, AGEs contribute to the mechanisms responsible for the development of complications in diabetes. Formation of intracellular AGEs promotes violation in protein function, with their accumulation acting as an objective marker for the tissues glycation. The level of skin fluorescence observed in our studies is related to the degree of conventional glycation marker HbA1c as determined in vitro. It is important to note, however, that the standard measure of glycation using HbA1c characterizes the glycation processes that occur only in the short term (around 3 months). Changes in AGE content reflect long-term processes in the diabetic skin. Considering the long molecular lifetime of collagen and the stability of AGEs, it is possible to use skin fluorescence as a measure of the total impact of hyperglycemia throughout the course of life.

It is known that diabetes can lead to tissue hypoxia. ${ }^{72-74}$ In the event of hypoxia, the aerobic cellular respiration pathway is disrupted, and the mitochondrial NADH oxidation is slowed down. Additionally, the glycolysis pathway for NADH formation is activated. In this context, the accumulation of NADH may act as a sign of tissue hypoxia, with its contribution to the total fluorescence signal serving as a marker of total oxygen deficiency in tissues. Meanwhile, the processes of oxidative phosphorylation exhibit changes in the concentrations of NADH and FAD, which are very complex, and the dynamics of these changes may be nonlinear throughout the cell's lifetime. ${ }^{38,39}$ This should be taken into account when interpreting the data received and further researching in this area.

It is also shown that the accumulation of AGEs may suppress the synthesis of NO in endothelial cells. ${ }^{75-77}$ This may also explain the differences in perfusion response to various stimuli. Basal level does not statistically differ among the compared groups. However, the proposed functional stimulation in the form of thermal tests can detect and distinguish between microvascular abnormalities in diabetic patients. The perfusion increase during a local heating test occurs mainly due to two mechanisms. The heating of skin to $34^{\circ} \mathrm{C}$ to $35^{\circ} \mathrm{C}$ results in activation of peptidergic nerve fibers. This happens due to the activation of thermosensitive TRPV1 receptors. ${ }^{78}$ The vasodilation caused by heating skin to $42^{\circ} \mathrm{C}$ is associated with a release of NO from vascular endothelium. ${ }^{79,80}$ Topographically, both

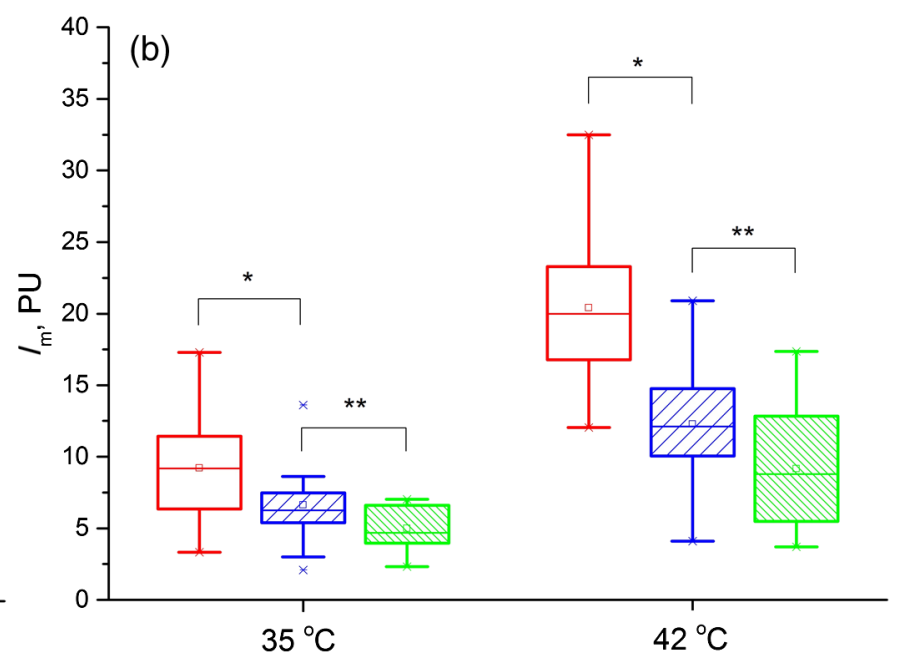

Fig. 4 Comparison of parameters among control (red empty bars), diabetic (blue loose shading), and diabetic with ulcers (green tight shading) groups: (a) the normalized fluorescence amplitude and (b) the average perfusion in the stages of heating to $35^{\circ} \mathrm{C}$ and $42^{\circ} \mathrm{C}$. In each box, the central line is the median of the group, while the edges are the 25 th and 75 th percentiles. 
Table 3 Sensitivity and specificity for the first classification rule.

\begin{tabular}{lcccccccc} 
Parameter & $\mathrm{AF}_{460}$ & $\mathrm{AF}_{525}$ & $I_{m}^{35^{\circ} \mathrm{C}}$ & $I_{m}^{42^{\circ} \mathrm{C}}$ & $\mathrm{AF}_{460}, I_{m}^{35^{\circ} \mathrm{C}}$ & $\mathrm{AF}_{460}, I_{m}^{42^{\circ} \mathrm{C}}$ & $\mathrm{AF}_{525}, I_{m}^{35^{\circ} \mathrm{C}}$ & $\mathrm{AF}_{525}, I_{m}^{42^{\circ} \mathrm{C}}$ \\
\hline Sensitivity & 0.64 & 0.77 & 0.77 & 0.88 & 0.75 & 0.92 & 0.87 & 0.90 \\
Specificity & 0.7 & 0.79 & 0.62 & 0.81 & 0.77 & 0.90 & 0.77 & 0.83 \\
\hline
\end{tabular}

arterioles and capillaries are involved in local heating. All aspects of the microvasculature and tissue systems undergo dysfunction during diabetes, including the vascular endothelial and perivascular nerve fibers. Therefore, the thermal test is pathogenically justified for diagnosing these disorders during diabetes. A reduction in perfusion growth when skin is heated to $35^{\circ} \mathrm{C}$ acts as an objective criterion of sensory nerve fiber dysfunction, which in turn serves as a component of diabetic neuropathy. The reduction of perfusion growth in people with diabetes compared with the control group during skin heating to $42^{\circ} \mathrm{C}$ reflects a deficit of endothelium-dependent vasodilation mechanisms (presumably NO-induced).

\subsection{Data Analysis}

The analyzed parameters of normalized skin fluorescence amplitudes and perfusion are proposed for the synthesis of the decision rule. As stated above, these parameters satisfy the principles of statistical independence, as well as the significance of the differences of their values, calculated for the patient and control groups. The discriminant function is synthesized in such a way as to provide high sensitivity while providing excellent specificity. Table 3 summarizes the sensitivity and specificity for the first classification rule (the control group is compared with a group of patients without ulcers) for a different combination of measured parameters. As can be seen from the table, the lowest level of error is obtained with the combination of fluorescence intensity at an excitation wavelength of $365 \mathrm{~nm}$ and the level of stimulation of microcirculatory perfusion at $42^{\circ} \mathrm{C}$.

For the second classification rule for the separation of the diabetic group and diabetic group with ulcers, a sensitivity and specificity of 0.86 and 0.85 , respectively, were obtained.

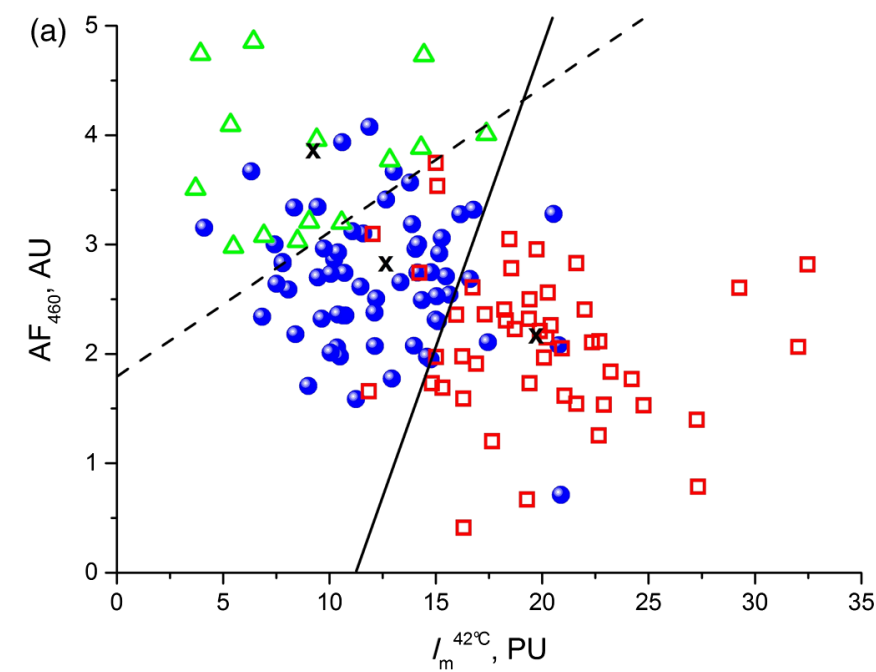

For better combinations of sensitivity and specificity, Fig. 5(a) shows the scatter plot of the experimental data with applied discriminant lines that divide the experimental points into three groups (healthy, diabetic, and diabetic with ulcers). The immediate diagnostic criterion, with the aid of the discriminant functions $\left(D_{1}\right.$ and $\left.D_{2}\right)$, allows for the relation of a newly measured subject to one of the three groups:

$$
\left\{\begin{array}{l}
D 1=-2.55-0.45 \mathrm{AF}_{460}+0.23 I_{m}^{42^{\circ} \mathrm{C}}, \\
D 2=-1.8+1.16 \mathrm{AF}_{460}-0.18 I_{m}^{42^{\circ} \mathrm{C}} .
\end{array}\right.
$$

It can be seen from Fig. 5(a) that the shift toward the top-left characterizes the deterioration of the patient's condition and increase in the risk of development of foot ulcers.

Figure 5(b) shows the ROC curves calculated for the obtained discriminant functions. To compare the quality of different classifying rules, it is convenient to use the integral characteristic AUC-area under curve. In our case, for both classification rules-AUC $=0.93$. This indicator indicates a high level of quality of the classifier.

Thus, the skin fluorescence and level of tissue blood perfusion during a local heating test can act as markers for various stages of diabetes, beginning with early disease, to the advancement and development of trophic ulcers.

\section{Conclusion}

The obtained experimental results confirm the prospects of the development of new methods for evaluating metabolic shifts in diabetes mellitus. The proposed method could be a marker of diabetic complications. It can also be used to assess therapeutic

Fig. 5 (a) The scatter plot with applied discriminant lines, obtained by linear discriminant analysis method and (b) ROC-curves for assessing the effectiveness of the classifiers. The healthy group is shown by squares, the diabetic group by circles, and the diabetic group with ulcers by triangles. For the ROC curve: dot line: control group versus diabetic group 1; solid line: diabetic group 1 versus diabetic group 2. 
interventions aimed at preventing or reversing the progression of these diabetic complications.

Modeling and experimental results of this study confirm the high sensitivity of optical noninvasive methods (laser Doppler flowmetry and fluorescence spectroscopy) when detecting violations of biological tissue in type 2 diabetes mellitus. Both approaches, either individually or in combination, may have important clinical implications as they may help to identify patients at risk of developing future problems within their lower limbs.

Improvement of methodological support of optical noninvasive diagnosis (convenient and completely safe for the patient) will make a significant contribution to the fight against diabetes, which is very relevant given the prevalence of the disease.

There is also further potential to expand the number of clinical investigations and improve the methodology. This will eventually allow the methods in question to be introduced into the practice of the attending physician.

A promising direction for future research within this field is the differentiation of AGEs and NADH and FAD contributions to the resultant fluorescence signal. This would allow for separate studies into the possible pathways of biological tissue violation: by oxidative phosphorylation and carbohydrate metabolism.

\section{Disclosures}

No potential conflicts of interest relevant to this article were reported.

\section{Acknowledgments}

This work was supported by funding from the European Union's Horizon 2020 research and innovation programme under grant agreement No. 703145. The authors also acknowledge the support of the Academy of Finland (grants: 296408 and 290596) as well as a grant of the President of the Russian Federation for state support of young Russian scientists No. MK-7168.2016.8. Special thanks are extended to doctors Alimicheva E.A., Masalygina G.I., and Muradyan V.F. of the Orel Regional Clinical Hospital for providing useful advice and help.

\section{References}

1. IDF Diabetes Atlas, 7th ed., International Diabetes Federation, Brussels, Belgium (2015).

2. J. B. Rice et al., "Burden of diabetic foot ulcers for medicare and private insurers," Diabetes Care 37(3), 651-658 (2014).

3. S. Zimny et al., "Early detection of microcirculatory impairment in diabetic patients with foot at risk," Diabetes Care 24(10), 1810-1814 (2001).

4. R. L. Greenman et al., "Early changes in the skin microcirculation and muscle metabolism of the diabetic foot," Lancet 366(9498), 1711-1717 (2005).

5. J. Cobb and D. Claremont, "Noninvasive measurement techniques for monitoring of microvascular function in the diabetic foot," Int. J. Lower Extremity Wounds 1(3), 161-169 (2002).

6. P. Zhang et al., "Global epidemiology of diabetic foot ulceration: a systematic review and meta-analysis dagger," Ann. Med. 49(2), 106-116 (2017).

7. J. Z. M. Lim, N. S. L. Ng, and C. Thomas, "Prevention and treatment of diabetic foot ulcers," J. R. Soc. Med. 110(3), 104-109 (2017).

8. M. E. Ahmadi et al., "Neuropathic arthropathy of the foot with and without superimposed osteomyelitis: MR imaging characteristics," Radiology 238(2), 622-631 (2006).

9. D. S. Chatha, P. M. Cunningham, and M. E. Schweitzer, "MR imaging of the diabetic foot: diagnostic challenges," Radiol. Clin. North Am. 43(4), 747-759 (2005).

10. N. Prandini et al., "Nuclear medicine imaging of bone infections," Nucl. Med. Сотmun. 27(8), 633-644 (2006).
11. D. T. Ubbink, "Toe blood pressure measurements in patients suspected of leg ischaemia: a new laser Doppler device compared with photoplethysmography," Eur. J. Vasc. Endovasc. Surg. 27(6), 629-634 (2004).

12. J. C. de Graaff et al., "The usefulness of a laser Doppler in the measurement of toe blood pressures," J. Vasc. Surg. 32(6), 1172-1179 (2000).

13. L. Ryden et al., "ESC guidelines on diabetes, pre-diabetes, and cardiovascular diseases developed in collaboration with the EASD: the task force on diabetes, pre-diabetes, and cardiovascular diseases of the European Society of Cardiology (ESC) and developed in collaboration," Eur. Heart J. 34(39), 3035-3087 (2013).

14. N. M. J. Hanssen et al., "Associations between the ankle-brachial index and cardiovascular and all-cause mortality are similar in individuals without and with type 2 diabetes: nineteen-year follow-up of a population-based cohort study," Diabetes Care 35(8), 1731-1735 (2012).

15. J. Haraden and C. Jaenicke, "Correlation of preoperative ankle-brachial index and pulse volume recording with impaired saphenous vein incisional wound healing post coronary artery bypass surgery," J. Vasc. Nurs. 24(2), 35-45 (2006).

16. C. Hoyer, J. Sandermann, and L. J. Petersen, "The toe-brachial index in the diagnosis of peripheral arterial disease," J. Vasc. Surg. 58(1), 231238 (2013).

17. K. Huang et al., "The correlation between transcutaneous oxygen tension and microvascular complications in type 2 diabetic patients," J. Diabetes Complications 31(5), 886-890 (2017).

18. P. Poredoš, "Validity of transcutaneous oxygen measurement in peripheral arterial disease," Int. Angiol. 36(4), 392-393 (2017).

19. C. E. Fife et al., "Transcutaneous oximetry in clinical practice: consensus statements from an expert panel based on evidence," Undersea Hyperbaric Med. 36(1), 43-53 (2009).

20. K. A. Arsenault et al., "The use of transcutaneous oximetry to predict healing complications of lower limb amputations: a systematic review and meta-analysis," Eur. J. Vasc. Endovasc. Surg. 43(3), 329-336 (2012).

21. A. R. Berendt and B. Lipsky, "Is this bone infected or not? Differentiating neuro-osteoarthropathy from osteomyelitis in the diabetic foot," Curr. Diabetes Rep. 4(6), 424-429 (2004).

22. A. J. Boulton and L. Vileikyte, "The diabetic foot: the scope of the problem," J. Fam. Pract. 49(11 Suppl.), S3-S8 (2000).

23. S.-J. Lin and L. Guarente, "Nicotinamide adenine dinucleotide, a metabolic regulator of transcription, longevity and disease," Curr. Opin. Cell Biol. 15(2), 241-246 (2003).

24. J. H. Hwang et al., "Pharmacological stimulation of NADH oxidation ameliorates obesity and related phenotypes in mice," Diabetes 58(4), 965-974 (2009).

25. Y. Ido, C. Kilo, and J. R. Williamson, "Cytosolic NADH/NAD+, free radicals, and vascular dysfunction in early diabetes mellitus," Diabetologia 40(Suppl. 2), S115-S117 (1997).

26. A. A. Heikal, "Intracellular coenzymes as natural biomarkers for metabolic activities and mitochondrial anomalies," Biomarkers Med. 4(2), 241-263 (2010).

27. D. Pouli et al., "Imaging mitochondrial dynamics in human skin reveals depth-dependent hypoxia and malignant potential for diagnosis," Sci. Transl. Med. 8(367), 367ra169 (2016).

28. N. Rajaram et al., "Pilot clinical study for quantitative spectral diagnosis of non-melanoma skin cancer," Lasers Surg. Med. 42(10), 876-887 (2010).

29. M. Balu et al., "In vivo multiphoton NADH fluorescence reveals depthdependent keratinocyte metabolism in human skin," Biophys. J. 104(1), 258-267 (2013).

30. C. Li et al., "Multiphoton microscopy of live tissues with ultraviolet autofluorescence," IEEE J. Sel. Top. Quantum Electron. 16(3), 516523 (2010).

31. A. Mayevsky and B. Chance, "Oxidation-reduction states of NADH in vivo: from animals to clinical use," Mitochondrion 7(5), 330-339 (2007).

32. A. Mayevsky and G. G. Rogatsky, "Mitochondrial function in vivo evaluated by NADH fluorescence: from animal models to human studies," Am. J. Physiol. Cell Physiol. 292(2), C615-C640 (2007).

33. E. T. Obi-Tabot et al., "Changes in hepatocyte NADH fluorescence during prolonged hypoxia," J. Surg. Res. 55(6), 575-580 (1993).

34. F. Bartolome and A. Y. Abramov, "Measurement of mitochondrial NADH and FAD autofluorescence in live cells," Methods Mol. Biol. 1264, 263-270 (2015). 
35. K. A. Foster, C. J. Beaver, and D. A. Turner, "Interaction between tissue oxygen tension and NADH imaging during synaptic stimulation and hypoxia in rat hippocampal slices," Neuroscience 132(3), 645-657 (2005).

36. I. J. Rampil, L. Litt, and A. Mayevsky, "Correlated, simultaneous, multiple-wavelength optical monitoring in vivo of localized cerebrocortical NADH and brain microvessel hemoglobin oxygen saturation," J. Clin. Monit. 8(3), 216-225 (1992).

37. G. Papayan, N. Petrishchev, and M. Galagudza, "Autofluorescence spectroscopy for NADH and flavoproteins redox state monitoring in the isolated rat heart subjected to ischemia-reperfusion," Photodiagn. Photodyn. Ther. 11(3), 400-408 (2014).

38. M. Aldakkak et al., "Modulation of mitochondrial bioenergetics in the isolated guinea pig beating heart by potassium and lidocaine cardioplegia: implications for cardioprotection," J. Cardiovasc. Pharmacol. 54(4), 298-309 (2009).

39. J. An et al., "Warm ischemic preconditioning improves mitochondrial redox balance during and after mild hypothermic ischemia in guinea pig isolated hearts," Am. J. Physiol. Heart Circ. Physiol. 288(6), H2620H2627 (2005).

40. P. Gkogkolou and M. Bohm, "Advanced glycation end products: key players in skin aging?" Derm. Endocrinol. 4(3), 259-270 (2012).

41. R. Meerwaldt et al., "Simple non-invasive assessment of advanced glycation endproduct accumulation," Diabetologia 47(7), 1324-1330 (2004).

42. K. C. Tan et al., "Advanced glycation end products and endothelial dysfunction in type 2 diabetes," Diabetes Care 25(6), 1055-1059 (2002).

43. E. G. Gerrits et al., "Skin autofluorescence: a tool to identify type 2 diabetic patients at risk for developing microvascular complications," Diabetes Care 31(3), 517-521 (2008).

44. D. R. Sell et al., "Pentosidine: a molecular marker for the cumulative damage to proteins in diabetes, aging, and uremia," Diabetes Metab. Res. Rev. 7(4), 239-251 (1991).

45. D. R. Sell et al., "Pentosidine formation in skin correlates with severity of complications in individuals with long-standing IDDM," Diabetes 41(10), 1286-1292 (1992).

46. M. Takahashi et al., "Direct measurement of crosslinks, pyridinoline, deoxypyridinoline, and pentosidine, in the hydrolysate of tissues using high-performance liquid chromatography," Anal. Biochem. 232(2), 158-162 (1995).

47. V. V. Tuchin, Handbook of Photonics for Biomedical Science, CRC Press, Boca Raton, Florida (2010).

48. E. A. Zherebtsov et al., "Combined use of laser Doppler flowmetry and skin thermometry for functional diagnostics of intradermal finger vessels," J. Biomed. Opt. 22(4), 040502 (2017).

49. A. I. Zherebtsova et al., "Study of the functional state of peripheral vessels in fingers of rheumatological patients by means of laser Doppler flowmetry and cutaneous thermometry measurements," Proc. SPIE 9917, 99170M (2016).

50. N.-T. Yu et al., "Development of a noninvasive diabetes screening device using the ratio of fluorescence to Rayleigh scattered light," J. Biomed. Opt. 1(3), 280-288 (1996).

51. V. V. Dremin et al., "The development of attenuation compensation models of fluorescence spectroscopy signals," Proc. SPIE 9917, 99170Y (2016).

52. A. V. Dunaev et al., "Individual variability analysis of fluorescence parameters measured in skin with different levels of nutritive blood flow," Med. Eng. Phys. 37(6), 574-583 (2015).

53. S. Beckert et al., "The impact of the micro-lightguide $\mathrm{O} 2 \mathrm{C}$ for the quantification of tissue ischemia in diabetic foot ulcers," Diabetes Care 27(12), 2863-2867 (2004).

54. V. V. Dremin et al., "The blood perfusion and NADH/FAD content combined analysis in patients with diabetes foot," Proc. SPIE 9698, 969810 (2016).

55. A. V. Dunaev et al., "Novel measure for the calibration of laser Doppler flowmetry devices," Proc. SPIE 8936, 89360D (2014).

56. International Commission on Non-Ionizing Radiation Protection, "Guidelines on limits of exposure to ultraviolet radiation of wavelengths between $180 \mathrm{~nm}$ and $400 \mathrm{~nm}$ (incoherent optical radiation)," Health Phys. 87(2), 171-186 (2004).

57. J. M. Johnson and D. W. Proppe, "Cardiovascular adjustments to heat stress," in Comprehensive Physiology, John Wiley \& Sons, Inc., New York (2010).
58. I. Fredriksson et al., "Reduced arteriovenous shunting capacity after local heating and redistribution of baseline skin blood flow in type 2 diabetes assessed with velocity-resolved quantitative laser Doppler flowmetry," Diabetes 59(7), 1578-1584 (2010).

59. Y.-K. Jan et al., "Skin blood flow response to locally applied mechanical and thermal stresses in the diabetic foot," Microvasc. Res. 89, 40-46 (2013).

60. M. Thoresen and L. Walloe, "Skin blood flow in humans as a function of environmental temperature measured by ultrasound," Acta Physiol. Scand. 109(3), 333-341 (1980).

61. L. Vanggaard et al., "Thermal responses to whole-body cooling in air with special reference to arteriovenous anastomoses in fingers," Clin. Physiol. Funct. Imaging 32(6), 463-469 (2012).

62. D. Fuchs et al., "The association between diabetes and dermal microvascular dysfunction non-invasively assessed by laser Doppler with local thermal hyperemia: a systematic review with meta-analysis," Cardiovasc. Diabetol. 16, 11 (2017).

63. E. A. Zherebtsov et al., "The influence of local pressure on evaluation parameters of skin blood perfusion and fluorescence," Proc. SPIE 10336, 1033608 (2017).

64. A. V. Dunaev et al., "Substantiation of medical and technical requirements for noninvasive spectrophotometric diagnostic devices," $J$. Biomed. Opt. 18(10), 107009 (2013).

65. I. E. Rafailov et al., "Computational model of bladder tissue based on its measured optical properties," J. Biomed. Opt. 21(2), 025006 (2016).

66. V. V. Dremin and A. V. Dunaev, "How the melanin concentration in the skin affects the fluorescence-spectroscopy signal formation," J. Opt. Technol. 83(1), 43-48 (2016).

67. C. Zhu and Q. Liu, "Review of Monte Carlo modeling of light transport in tissues," J. Biomed. Opt. 18(5), 050902 (2013).

68. G. I. Petrov et al., "Human tissue color as viewed in high dynamic range optical spectral transmission measurements," Biomed. Opt. Express 3(9), 2154-2161 (2012).

69. I. V. Meglinski and S. D. Matcher, "Analysis of the spatial distribution of detector sensitivity in a multilayer randomly inhomogeneous medium with strong light scattering and absorption by the Monte Carlo method," Opt. Spectrosc. 91(4), 654-659 (2001).

70. I. V. Meglinsky and S. J. Matcher, "Modelling the sampling volume for skin blood oxygenation measurements," Med. Biol. Eng. Comput. 39(1), 44-50 (2001).

71. I. Meglinski and A. Doronin, "Monte Carlo modeling of photon migration for the needs of biomedical optics and biophotonics," in Advanced Biophotonics, Chapter 1, pp. 1-72, Taylor \& Francis (2013).

72. L. Xi, C.-M. Chow, and X. Kong, "Role of tissue and systemic hypoxia in obesity and type 2 diabetes," J. Diabetes Res. 2016, 1-3 (2016).

73. H. Thangarajah et al., "The molecular basis for impaired hypoxiainduced VEGF expression in diabetic tissues," Proc. Natl. Acad. Sci. U. S. A. 106(32), 13505-13510 (2009).

74. J. Ditzel and E. Standl, "The problem of tissue oxygenation in diabetes mellitus," Acta Med. Scand. Suppl. 578, 59-68 (1975).

75. R. Bucala, K. J. Tracey, and A. Cerami, "Advanced glycosylation products quench nitric oxide and mediate defective endothelium-dependent vasodilatation in experimental diabetes," J. Clin. Invest. 87(2), 432-438 (1991).

76. A. W. Stitt et al., "Elevated AGE-modified ApoB in sera of euglycemic, normolipidemic patients with atherosclerosis: relationship to tissue AGEs," Mol. Med. 3(9), 617-627 (1997).

77. U. Chakravarthy et al., "Constitutive nitric oxide synthase expression in retinal vascular endothelial cells is suppressed by high glucose and advanced glycation end products," Diabetes 47(6), 945-952 (1998).

78. D. P. Stephens et al., "The influence of topical capsaicin on the local thermal control of skin blood flow in humans," Am. J. Physiol. Regul. Integr. Comp. Physiol. 281(3), R894-R901 (2001).

79. C. T. Minson, L. T. Berry, and M. J. Joyner, "Nitric oxide and neurally mediated regulation of skin blood flow during local heating," J. Appl. Physiol. 91(4), 1619-1626 (2001).

80. D. L. Kellogg, Jr., J. L. Zhao, and Y. Wu, "Roles of nitric oxide synthase isoforms in cutaneous vasodilation induced by local warming of the skin and whole body heat stress in humans," J. Appl. Physiol. 107(5), 14381444 (2009). 
Viktor V. Dremin received his MSc degree from Orel State University, Orel, Russia, in 2013. He is now finishing his PhD at Orel State University. Currently, he is a researcher in Biomedical Photonics Laboratory of the University Clinic. His research interests include methodological provision and instrumentation of fluorescence spectroscopy of biological tissue in vivo, modeling of optical radiation propagation in biological tissues.

Evgeny A. Zherebtsov received his MSc and $\mathrm{PhD}$ degrees from Orel State University, Orel, Russia, in 2010 and 2013, respectively. Currently, he is a Marie Curie fellow at Aston University, Birmingham, United Kingdom. His research interests include biomedical imaging, optical tools for assessment of metabolic activity of biological tissues, modeling of optical radiation propagation in biological tissues. He is member of SPIE.

Victor V. Sidorov received his PhD from the Scientific-Production Enterprise "Astrophisica," Moscow, USSR in 1989. He is a CEO of SPE "LAZMA," Moscow, Russia. His research interests include design of methods and laser equipment for noninvasive optical diagnostics.

Alexander I. Krupatkin received $\mathrm{PhD}$ and DMSc degree in the Central Institute of Traumatology and Orthopedics (CITO), Moscow, Russia, in 1988 and 1999, respectively. He is the leading researcher in the Department of Functional Diagnosis, CITO. His research interests include clinical physiology of microcirculation, oscillations in microvascular-tissue systems, control of blood and lymph flow.

Irina N. Makovik received her MSc degree from Orel State University, Orel, Russia, in 2013. She is a PhD student of the department "instrumentation, metrology and certification" at Orel State University. She is an engineer researcher in Biomedical Photonics Laboratory of the University Clinic in February 2014. Her research focuses on the application of optics and photonics in clinical practice. He is member of SPIE.

Angelina I. Zherebtsova received her MSc and $\mathrm{PhD}$ degrees from Orel State University, Orel, Russia, in 2011 and 2016, respectively. Currently, she is a researcher in Biomedical Photonics Laboratory of the University Clinic, Orel, Russia. Her research interests include noninvasive diagnostics of functional state of peripheral vessels and investigation of microcirculation blood flow and skin temperature changes during functional tests.

Elena V. Zharkikh received her BSc degree from Orel State University, Orel, Russia, in 2017 and entered her MSc program there. Currently, she is a researcher in Biomedical Photonics Laboratory of the University Clinic, Orel, Russia. Her research interests include methodological provision and instrumentation of fluorescence spectroscopy of biological tissue in vivo and investigation of blood microcirculation changes during different functional tests. He is member of SPIE.

Elena V. Potapova received her MSc and $\mathrm{PhD}$ degrees from Orel State University, Orel, Russia, in 2004 and 2008, respectively. Currently, she is a researcher in Biomedical Photonics Laboratory of the University Clinic, Orel, Russia. Her research interests include methodological provision and instrumentation of fluorescence spectroscopy and diffuse reflectance spectroscopy of biological tissue in vivo.

Andrey V. Dunaev received his MSc and PhD degrees from Orel State University, Orel, Russia, in 1999 and 2002, respectively. Currently, he is a head in Biomedical Photonics Laboratory of the University Clinic, Orel, Russia and Orel State University, Orel, Russia. His research interests include metrological, methodological, and instrumentation support of noninvasive optical diagnostics. He is a member of SPIE and expert of the Russian Academy of Sciences.

Alexander A. Doronin is a postdoctoral associate in computer science. His research interests are interdisciplinary and lie at the interface between computer science, physics, optics and biophotonics focusing on physically-based rendering, development of realistic material models, Monte Carlo modeling of light transport in turbid media, color perception, translucency, appearance and biomedical visualization.

Alexander V. Bykov is a senior research fellow/adjunct professor in the Optoelectronics and Measurement Techniques Unit, University of Oulu, Finland. He received his PhD from Lomonosov Moscow State University, Russia, in 2008. He received his DSc (Tech.) degree from the Faculty of Technology, University of Oulu, Finland, in 2010. He is an author and coauthor of over 80 papers in peer-reviewed scientific journals, international conference proceedings and book chapters.

llya E. Rafailov obtained his MSc degree in neuroscience from Kings College University, London, UK. He is now finishing his $\mathrm{PhD}$ at Aston University, Birmingham, UK, concentrating on biomedical diagnostics through photonics technologies. His primary interests include noninvasive diagnostics of conditions, such as cancer and cardiovascular diseases.

Karina S. Litvinova received her MSc degree in engineering from Moscow Aviation University, Moscow, Russia and her PhD in biophysics and physiology from the Institute for Biomedical Problems of Russian Academy of Sciences, Moscow, Russia. Her research areas are biomedicaloptics and biophotonics, fluorescence spectroscopy.

Sergei G. Sokolovski received his MSc degree from Byelorussian State University in 1989 and his PhD from the National Academy of Sciences of Belarus in 1993. Work in the interdisciplinary field of biophotonics, noninvasive laser diagnostics. He has 27 year of experience in photobiology, biophysics, and cell molecular biology. His academia and industrial collaborating work both national and international has resulted in over 49 publications (965 citations) including three chapters in invited books, two patents (USA 13/ 164,452, and GB 1520886.1), and eight successful grant applications.

Edik U. Rafailov received his PhD from loffe Institute. He established new group in 2005 and his Optoelectronics and Biomedical Photonics Group moved to Aston University in 2014. He has authored and coauthored over 350 articles in refereed journals and conference proceedings. His current research interests include high-power $\mathrm{CW}$, ultrashortpulse lasers, generation of UV/visible/IR/MIR and THz radiation, nanostructures, nonlinear and integrated optics, and biophotonics. 